Case report

\title{
Severe dysphagia due to a huge epiphrenic diverticulum: long-term treatment with balloon dilation and botulinum toxin injection: a case report
} Panagiotis Katsinelos, Grigoris Chatzimavroudis*, Kostas Fasoulas, Ioannis Pilpilidis, Georgia Lazaraki, Taxiarchis Katsinelos, Dimitris Tzilves, George Germanidis and Themistoklis Vasiliadis

Address: Department of Endoscopy and Motility Unit, "G. Gennimatas" General Hospital of Thessaloniki, Ethnikis Aminis 41, Thessaloniki, Greece

Email: GC* - gchatzimav@yahoo.gr

* Corresponding author

Received: 20 January 2009 Accepted: 9 March 2009 Published: II June 2009

Cases Journal 2009, 2:7418 doi: 10.4076/1757-1626-2-7418

This article is available from: http://casesjournal.com/casesjournal/article/view/74l8

(C) 2009 Katsinelos et al; licensee Cases Network Ltd.

This is an Open Access article distributed under the terms of the Creative Commons Attribution License (http://creativecommons.org/licenses/by/3.0),

which permits unrestricted use, distribution, and reproduction in any medium, provided the original work is properly cited.

\begin{abstract}
We herein describe the first case of a high elderly patient with severe dysphagia in solids and liquids, caused by a huge epiphrenic diverticulum, who was treated with combined therapy of balloon dilation and botulinum toxin injection. Due to comorbid associated diseases the patient was unsuitable to withstand surgical or laparoscopic intervention. Treatment with botulinum toxin injection at the region of lower esophageal sphincter was unsuccessful. Combined therapy with balloon dilatation and botulinum toxin injection at the compressed part of esophageal lumen by the diverticulum resulted in improvement in dysphagia and malnutrition. During the long-term follow-up the patient developed symptomatic relapses, successfully treated by subsequent combined therapy resulting in longer-lasting symptom relief.
\end{abstract}

\section{Introduction}

Epiphrenic diverticula (ED) are typically located 4 to $8 \mathrm{~cm}$ above the cardia, comprising about $10 \%$ of all esophageal diverticula [1]. They usually project from the right posterior wall of the esophagus and may be single or multiple. Despite ED are usually asymptomatic, especially when they are small, the enlargement of their size can produce symptoms such as dysphagia, regurgitation of undigested food, retrosternal discomfort, halitosis, and weight loss $[1,2]$. Reported complications include the occurrence of squamous cell carcinoma within the diverticula, hemorrhage, food impaction, bezoars formation and esophageal lumen obstruction [1-4].

We herein present the first case of a high elderly patient with severe dysphagia in solids and liquids, caused by a huge epiphrenic diverticulum, who was unsuitable for surgery due to comorbid diseases and who was treated with combined therapy of balloon dilation and botulinum toxin (BTX) injection. 


\section{Case presentation}

An 88-year-old man with chronic obstructive pulmonary disease and heart failure was referred to our department because he presented severe dysphagia in solid and liquid food during the last 6 months, leading to malnutrition. Clinical examination revealed an emaciated man. Laboratory data showed $\mathrm{Hb}$ of $11.3 \mathrm{~g} / \mathrm{dl}$ (normal range: 13.5-16). Ht of $30 \%$ (normal range: $40-48 \%$ ), serum albumin of $1.9 \mathrm{~g} / \mathrm{dl}$ (normal range: $3.5-5$ ) and normal liver and renal biochemistry. Esophagogram demonstrated a huge epiphrenic diverticulum in the posterior-lateral wall. Upper gastrointestinal endoscopy disclosed food debris and fluids in the esophagus. After aspiration of fluids and removal of food debris, a huge diverticulum associated with stagnation's esophagitis was demonstrated (Figure 1). The lumen of the esophagus was compressed by the diverticulum, resulting in obstruction and dysphagia. Esophageal manometry revealed findings of non-specific motility disorder.

Due to patient's associated comorbid diseases and poor general condition, surgical or laparoscopic treatment was considered unsuitable. We tried to treat the patient with BTX injection (100 IU) in the lower esophageal sphincter, with unsuccessful results. Endoscopic treatment that would combine balloon dilation of compressed by the diverticulum esophageal lumen with simultaneous BTX

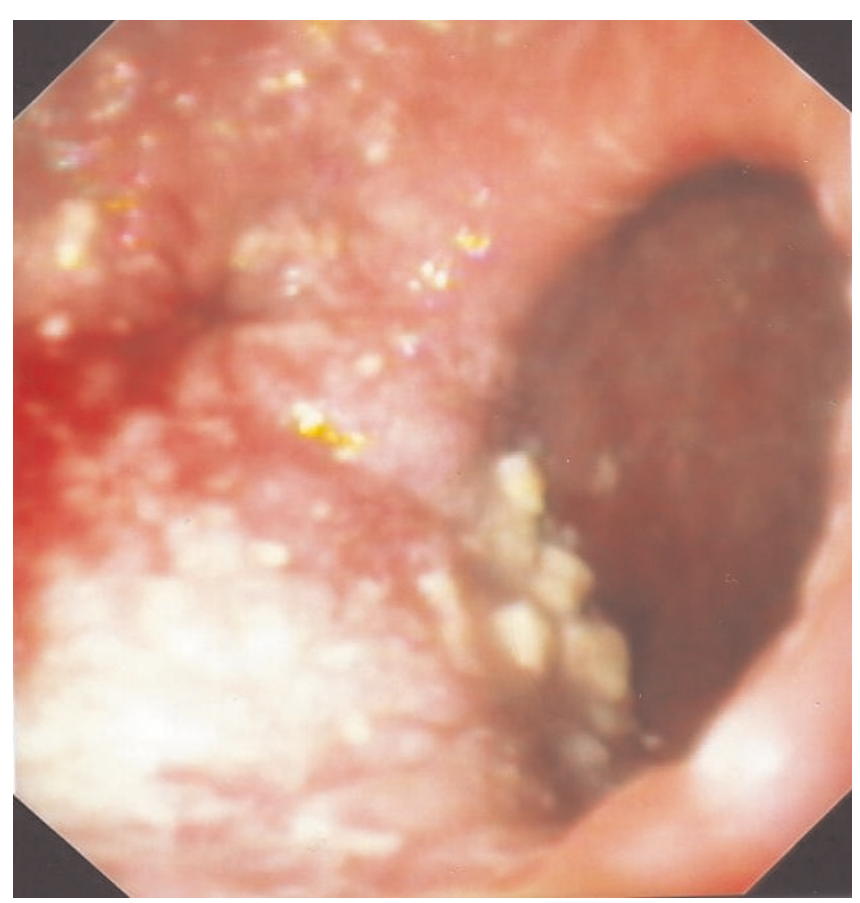

Figure I. Endoscopic view of a huge epiphrenic diverticulum associated with stagnation esophagitis.
(100 IU) injection in the proximal aspects of narrowest area of esophageal lumen was considered as an alternative treatment that could be applied in this high-risk patient.

After an extensive discussion with patient's relatives about benefits and complications of our suggested treatment, a consent form was signed by them. We dilated the compressed by the diverticulum esophageal lumen (Figure 2) with a through-the-scope balloon dilator (diameter $25 \mathrm{~mm}$, Microvasive, Boston Scientific, USA). The duration of dilation was $1 \mathrm{~min}$ and was repeated three times. The dilation was followed by BTX injection. BTX of 100 IU (Botox, Merc, Frankfurt, Germany) was dissolved in $4 \mathrm{ml}$ of normal saline $(25 \mathrm{IU} / \mathrm{ml})$ immediately before injection. Injections (1 ml each) via a sclerotherapy needle were introduced in the anterior, posterior and lateral parts of the proximal narrowest area of esophageal lumen (Figure 3). The patient showed a remarkable relief of his dysphagia since the first post-procedure week, resulting in weight gain and gradual improvement of walking. The beneficial effect of combined therapy lasted 8 weeks and thereafter the therapy was repeated every 6 to 8 months during last 28 months with very good results.

\section{Discussion}

Surgical or laparoscopic diverticulectomy combined with long esophagomyotomy is considered the treatment of

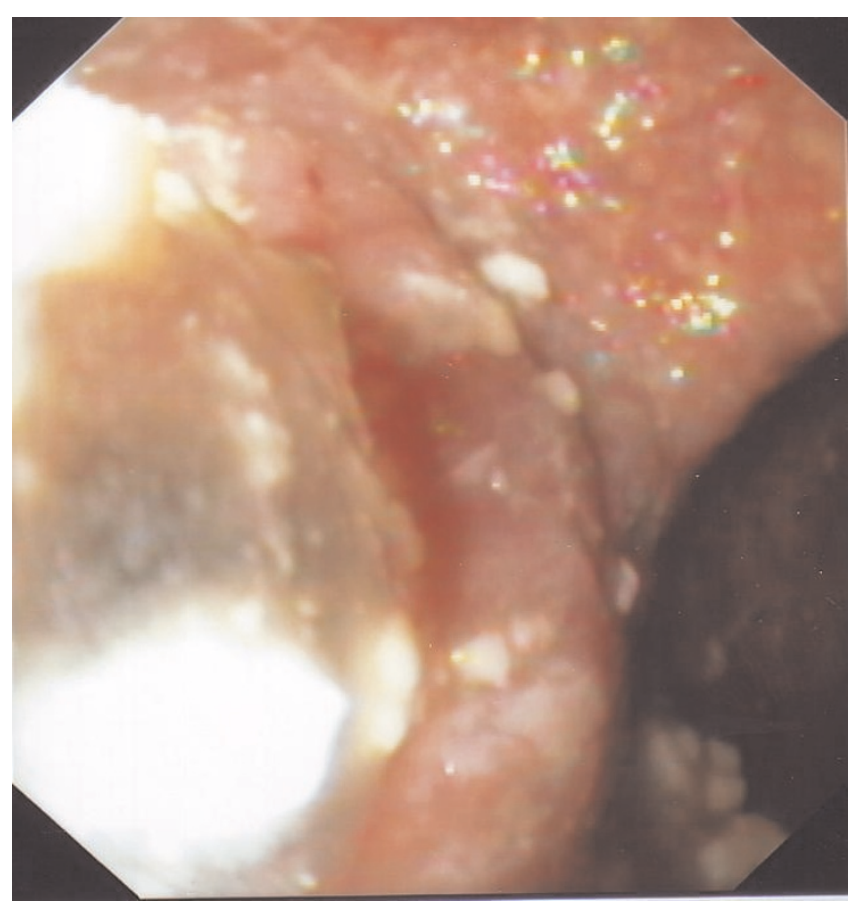

Figure 2. Endoscopic view showing the dilation of the compressed, by the diverticulum, esophageal lumen. 


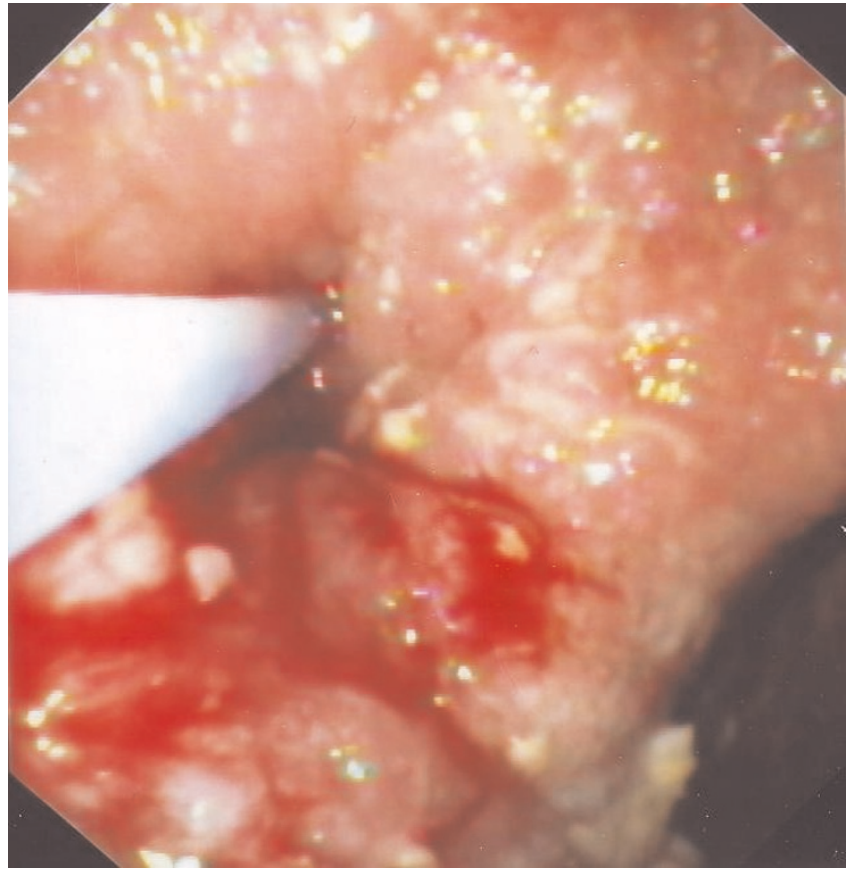

Figure 3. The presence of blood is due to BTX injection.

choice for symptomatic patients with ED $[2,3,5,6]$. However, for elderly patients with comorbid conditions, such as our patient, surgical or even laparoscopic intervention is associated with significant morbidity and mortality. As a consequence we preferred to start treatment with BTX injection in the lower esophageal sphincter. Despite BTX injection has been widely used in the treatment of achalasia [7], the reported role of the use of BTX in other dyskinetic diseases of the esophagus, such as diffuse esophageal spasm, isolated LES hypertension, dysphagia due to esophageal diverticula and other specific esophageal disorders, is very limited $[8,9]$.

There are only 3 reports on the use of BTX injection to resolve dysphagia due to midesophageal or epiphrenic diverticula. DeVault injected $80 \mathrm{IU}$ ( $1 \mathrm{ml}$ of $20 \mathrm{IU})$ through a $5 \mathrm{~mm}$ sclerotherapy needle into each of the four quadrants of the proximal aspect of the narrowed area distal to the diverticulum [10]. All 3 patients with midesophageal diverticula had remarkable improvement in their swallowing and remained in remission at 6-month follow-up examination. Unfortunately, there is no information on the long-term course of these patients. Pitchford and Price described a 75-year-old man with symptomatic epiphrenic diverticulum in whom they injected 100 IU of BTX into the LES, providing complete symptomatic relief [11]. Ten months later, however, the patient presented again with recurrent symptoms and underwent epiphrenic diverticulectomy. In a recent published paper we have described two elderly patients with dysphagia caused by large epiphrenic diverticula who were treated with BTX injected endoscopically at multiple sites in the region of the lower esophageal sphincter and esophageal wall near to the diverticulum, because they were unable to withstand surgical or laparoscopic intervention due to severe associated comorbid diseases [12]. Symptoms improved immediately and the beneficial effect of BTX remained for 5-6 months. During the long-term follow-up the patient developed symptomatic relapses, treated by subsequent BTX re-injections resulting in longer-lasting symptom relief.

In the patient described herein the initial use of BTX injection (100 IU) in the lower esophageal sphincter was not associated with symptom's relief. Our dilemma was to proceed to gastrostomy, enteral feeding, or to try an endoscopic treatment which combined the balloon dilation of compressed by the diverticulum esophageal lumen with simultaneous BTX (100 IU) injection in the proximal aspects of narrowest area of esophageal lumen. The relief of symptoms justified our therapeutic choice.

\section{Conclusions}

Combined treatment of BTX injection and balloon dilation of the narrowest area of the esophagus is a simple, safe and effective method, alternative to surgery, for high elderly patients with dysphagia due to ED and severe comorbid diseases.

\section{List of abbreviations}

\section{Competing interests}

The authors declare that they have no competing interests.

\section{Consent}

Written informed consent was obtained from the patient for publication of this case report and accompanying images. A copy of the written consent is available for review by the Editor-in-Chief of this journal.

\section{Author's contributions}

PK performed the endoscopic therapy and was contributor in writing the manuscript. GC and KF analyzed and interpreted the patient data and were contributors in writing the manuscript. IP, GL and DT reviewed the relative literature. GG and TV were contributors in revising the manuscript critically for important intellectual content. All authors read and approved the final manuscript. 


\section{References}

I. Clouse RE, Diamant NE. From Esophageal motor and sensory function and motor disorders of the esophagus. Sleisenger and Fordtran's Gastrointestinal and Liver disease. Edited by Feldman M, Freeman LS, Brandt LJ. Philadelphia, WB Saunders; 2006:855-904.

2. Hudspeth DA, Thorne MT, Conroy R, Pennell TC: Management of epiphrenic esophageal diverticula. A fifteen-year experience. Am Surg 1993, 59:40-42.

3. Benacci JC, Deschamps C, Trastek VF, Allen MS, Daly RC, Pairolero PC: Epiphrenic diverticulum: results of surgical treatment. Ann Thor Surg 1993, 55: I I09-I II3.

4. Lai ST, Hsu CP: Carcinoma arising from an epiphrenic diverticulum: a frequently misdiagnosed disease. Ann Thorac Cardiovasc Surg 2007, 13:1 I0-I I3.

5. Tedesco P, Fisichella PM, Way LW, Patti MG: Cause and treatment of epiphrenic diverticula. Am / Surg 2005, 190:89|-894.

6. Granderath FA, Pointner R: Laparoscopic transhiatal resection of giant epiphrenic esophageal diverticulum. Dis Esophagus 2007, 20:353-357.

7. Gui D, Rossi S, Runfola M, Magalini SC: Review article: Botulinum toxin in the therapy of gastrointestinal motility disorders. Aliment Pharmacol Ther 2003, 18: I- 16.

8. Miller LS, Parkman HP, Schiano TD, Cassidy MJ, Ter RB, Dabezies MA, Cohen S, Fisher RS: Treatment of symptomatic nonachalasia esophageal motor disorders with botulinum toxin injection at the lower esophageal sphincter. Dig Dis Sci 1996, 41:2025-2031.

9. Storr M, Allescher HD, Rosch T, Born P, Weigert N, Classen M: Treatment of symptomatic diffuse esophageal spasm by endoscopic injections of botulinum toxin: a prospective study with long-term follow-up. Gastrointest Endosc 200I, 54:754-759.

10. DeVault KR: Dysphagia from esophageal diverticulosis responding to Botulinum toxin injection. Am J Gastroenterol 1997, 92:895-897.

II. Pitchford TJ, Price PD: Laparoscopic Heller myotomy with epiphrenic diverticulectomy. JSLS 2003, 7:165-169.

12. Katsinelos P, Chatzimavroudis G, Zavos C, Pilpilidis I, Paroutoglou G, Kountouras ]: Long-term botulinum toxin treatment for dysphagia due to large epiphrenic diverticulum in elderly patients: a report of two cases. Dysphagia, in press.

\section{Do you have a case to share?}

Submit your case report today

- Rapid peer review

- Fast publication

- PubMed indexing

- Inclusion in Cases Database

\section{Any patient, any case, can teach us something}

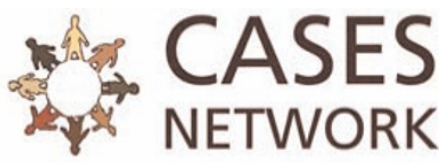

www.casesnetwork.com 